\title{
UMA ANÁLISE DE DESLOCAMENTO DE REBANHO BOVINO ATRAVÉS DOS MODELOS RANDOM WALK E BROWNIANO
}

\author{
COMPARING RANDOM WALKWITHBROWNIAN MOVEMENT TO SIMULATE BOVINE \\ HERD DISPLACEMENT
}

Tech, A.R.B. ${ }^{1,2}$, A.I.C. Arce ${ }^{2 A}$, A.C.S. Silva ${ }^{2 B}$ e E.J.X. Costa ${ }^{2 *}$

${ }^{1}$ Academia da Força Aérea Brasileira, AFA. Pirassununga/SP. Brasil. adriano.tech@usp.br ${ }^{2}$ Faculdade de Zootecnia e Engenharia de Alimentos. Universidade de São Paulo (FZEA-USP). Campus de Pirassununga. CEP 13635-900, SP. Brasil.Aacaid@usp.br; Banac_ss@yahoo.com.br. *Correspondencia: ernane@usp.br

\section{PaLAVRas ChaVe ADICIONAIS}

Comportamento do rebanho. Rede sem fio. Simulador.

\section{RESUMO}

O presente artigo simula o movimento de rebanho bovino através dos movimentos aleatórios random walk e browniano, procurando analisar qual desses prediz melhor o deslocamento de rebanhos bovinos, definindo parâmetros e descrevendo os algoritmos utilizados em ambos os movimentos e utilizando-os na construção dos eventos do simulador. Com a construção do simulador foi possível analisar os movimentos, bem como compreender que modelo de deslocamento melhor se aplica ao movimento de rebanho bovino. Também foi possível uma análise de desempenho espacial para a construção de redes de localização e identificação do rebanho através de sensores e redes sem fio.

\section{SUMMARY}

The present article simulate the movement of bovine herd through the aleatory movements random walk and brownian, trying to analyze which of those predicts the movement better of bovine herd displacement, defining parameters and describing the algorithms used in both movements and using them in the construction of the events of the simulator. With the construction of the simulator it was possible the analysis of the movements, besides the understand that model of displacement better if apply of bovine herd

Recibido: 14-9-07. Aceptado: 17-1-08.

\section{AdDitionAL KEYWORDS}

Behavior bovine herd. Wireless sensor network. Simulator.

displacement. Also it was possible an analysis of space acting for the construction of the location nets and identification of the herd through of wireless sensor network.

\section{INTRODUÇÃO}

Os processos de estudos espaciais e de gerenciamento animal em pastagens são elementos importantes e complexos que permitem o estudo de muitos fatores importantes, tanto na produtividade, como na qualidade. Sendo assim, o presente trabalho tem como premissa a confecção de um sistema computacional para simular o deslocamento do animal, usando um modelo baseado em variáveis reais do ambiente e do animal através de um modelo matemático permitindo a identificação e a localização do rebanho em pastagem.

Segundo Pressman (2004), a construção de um modelo, e conseqüentemente de um sistema, só é alcançada a partir da perfeita concepção do modelo real, fator esse, que permitirá compreender melhor o objeto em estudo e gerar o modelo virtual através de algoritmos e diagramas de fluxo de dados. 
Para que a construção do referido modelo fosse realizada, estudos sobre modelos de movimentos padronizados foram necessários. Tais modelos de movimentos devem ser simulados e implementados para que em um segundo momento, sejam comparados ao movimento descrito pelo rebanho.

Com a modelagem e identificação de elementos do comportamento de deslocamento do animal, foi possível desenvolver o modelo e identificar as funções ou objetos importantes (distância social, distância de fuga) para a construção do simulador, que permitiu também, a localização do animal e do próprio rebanho em tempo de execução durante o seu deslocamento. O sistema permitirá também a simulação do monitoramento do rebanho com a utilização de redes sem fio.

\section{ODESLOCAMENTOSOCIALDO BOVINO}

O estudo sobre o deslocamento social do bovino permitiu a modelagem do ambiente em estudo, através do mapeamento dos movimentos que foram implementados conforme os métodos citados. Desta forma, esse estudo identificou os requisitos necessários para a implementação dos elementos principais que fazem parte dos objetos desenvolvidos no simulador para descrição do movimento de deslocamento animal.

O comportamento de deslocamento do animal está inserido em seu bem-estar conforme caracterizado por Broom e Johnson (1993) como sendo o estado do organismo durante as suas tentativas de se ajustar ao seu ambiente.

Há muito que se discutir sobre o tema bem-estar-animal e conseqüentemente sobre o comportamento social de deslocamento do animal em relação as forças externas que atuam sobre ele, principalmente a relacionada ao seu manejo (Broom e Johnson, 1993).

Cientificamente, o termo comportamento animal é conhecer e respeitar a biologia dos animais em produção, melhorando o bemestar pode-se obter melhores resultados econômicos. Seja na eficiência do sistema ou na obtenção de produtos de melhor qualidade (Nadaleto e Marques, 2007) (Broom e Molento, 2004).

Alguns fatores são essenciais para a concepção do simulador, sendo a distância de conforto um elemento importante, pois acompanha o animal em todo o seu deslocamento, e uma vez quebrado esse elemento, o animal pode realizar movimentos inesperados na busca de uma nova área de estabilidade e segurança.

Segundo Costa et al. (2003) os bovinos possuem padrões de comportamentos através de hierarquias sociais, identificados através dos movimentos lineares ou de movimentos mais complexos. A figura 1 permite identificar esses movimentos e compreender melhor os modelos existentes podendo variar de acordo com algum tipo de situação não conhecida pelo rebanho.

O líder pode ser identificado de maneira simples, pois ele é o animal que inicia os movimentos e as atividades do grupo. Mas, deve-se observar que em um mesmo grupo existe a possibilidade de encontrar mais de um líder, sendo que, cada um, com uma função diferente (Costa et al., 2003).

Outro fator importante para a identificação de um modelo para a confecção do

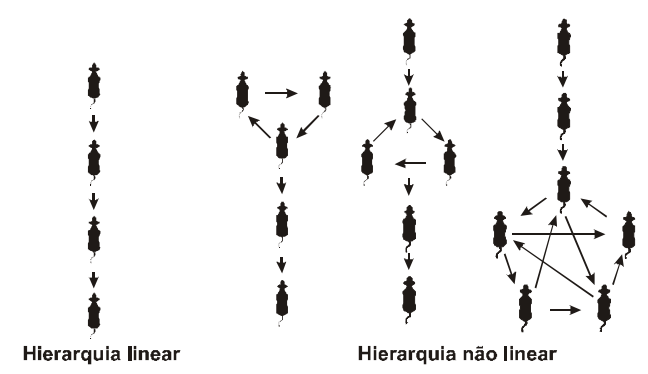

Figura 1. Tipos de movimentos. Adaptado de Herrera García et al. (2007). (Types of movements. Adapted from Herrera García et al. (2007)). 


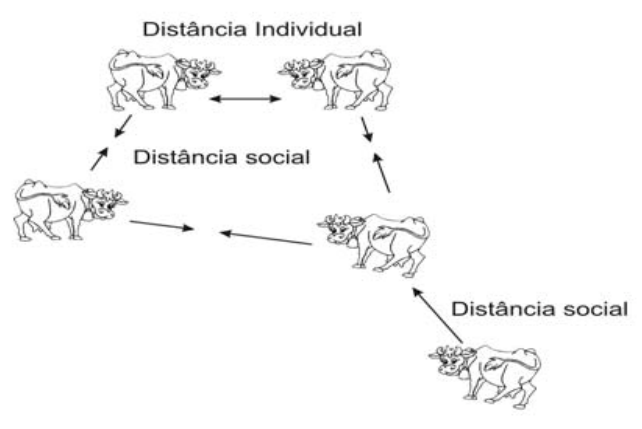

Figura 2. Distância social e individual. Adaptado de Herrera García et al. (2007). (Social and individual distance. Adapted from Herrera García et al. (2007)).

simulador, diz respeito às associações entre os elementos do grupo, sendo que, existirão associações mais fortes entre casais, entre indivíduos gêmeos e finalmente entre grupos filiais. Essa associação pode ser identificada pela distância existente entre animais vizinhos que normalmente é inferior a $5 \mathrm{~m}$ (Herrera García et al., 2007).

Segundo Herrera García et al. (2007) durante o deslocamento atuam duas forças opostas que afetam individualmente os membros do rebanho e cujo equilíbrio faz com que a distância entre os indivíduos do grupo seja uniforme, mantendo assim, uma distância de conforto segura e importante, principalmente à nível de deslocamento, o qual deve sempre ser realizado com muito cuidado e calma, para evitar que o animal e conseqüentemente o rebanho entre em um nível de stress que pode se tornar um fator complicador em relação ao deslocamento do grupo.

Outros fatores importantes em relação ao comportamento de deslocamento do animal estão relacionados as força coesivas e repulsivas (Herrera García et al., 2007). A primeira tende a unir os animais, pois esta relacionada aos diferentes níveis de associação (casais, gêmeos, grupos filiais), a segunda força realiza um processo contrário, pois tende a separar os animais e previne que os mesmos fiquem muito próxi- mos definindo assim, a zona de fuga dos indivíduos e distância crítica.

Através da figura 2, é possível verificar a atuação dessas forças que foram elementos importantes para a construção do simulador.

A distância individual ou distância mínima entre co-específicos tende a variar de acordo com o tipo de atividade feita pelo rebanho, mas podem-se identificar alguns valores pré-determinados como (Herrera García et al., 2007):

-Distância em relação ao descanso do animal $=$ de 2 a 3 metros, e

-Distância em relação a pastagem $=$ de 8 a 10 metros.

Outros tipos de distâncias podem ser observados, tais como:

-Distância social, distância máxima que se separa um indivíduo do grupo.

-Distância crítica, distância mínima que um indivíduo pode se aproximar.

Um outro fator importante a ser levado em consideração, diz respeito à área de conforto que é de aproximadamente $10 \mathrm{~m}^{2}$ por animal, conforme figura 3 (Grandin, 2000):

Uma vez, compreendido os principais

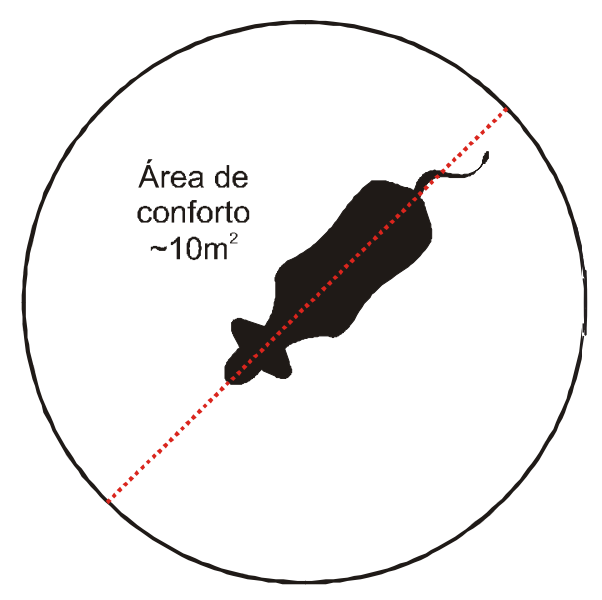

Figura 3 - Distância de conforto. Adaptado de Grandin (2000). (Distance of comfort. Adapted from Grandin (2000)). 
elementos a serem monitorados e implementados no simulador, passou-se a analisar a forma de movimento descrito por esses animais.

Esses elementos permitiram a construção e implementação do modelo, pois refletiam as características comportamentais do rebanho. Outros elementos serão descritos a seguir, para a construção e modelagem dos principais fatores que comporão os objetos em estudo, através da compreensão dos movimentos de deslocamento do animal.

\section{ESTUDO SOBRE AS CARACTERÍSTI- CAS DO MOVIMENTO ALEATÓRIO}

\section{MOVIMENTORANDOMWALK}

O movimento random walk descreve um algoritmo que usa números gerados de forma aleatória para calcular o deslocamento de uma partícula em uma rede simétrica. Pode-se compreender rede simétrica como sendo uma base de referência por onde as partículas se movimentarão.

Um exemplo desse tipo de rede simétrica pode ser compreendido através de um papel milimetrado, onde cada cruzamento das linhas identificaria uma posição a ser ocupada por uma partícula em um instante $\mathrm{t}+1$, sendo assim, o sistema seria capaz de predizer segundo um modelo matemático baseado em movimentos aleatórios as possíveis posições que a partícula ocuparia em sua próxima movimentação.

Segundo Sharov (1997) alguns estudos identificam que ao instanciar 50\% dos animais no mesmo lugar e variar os outros $50 \%$ em movimentos ou deslocamentos para a direita e para a esquerda, será possível verificar que após alguns passos $(n+1)$ ou em um determinado tempo $(\mathrm{t}+1)$, a distribuição desses animais tenderão a descrever uma distribuição normal, a qual pode ser verificar através da figura 4 , obtida pelo protótipo do simulador.

Através da figura 4 é possível visualizar o número de animais e o seu comportamento em relação ao tempo de deslocamento. Sendo assim, podemos representar uma distribuição normal através da seguinte fórmula (Sharov, 1997):

$$
p(x)=\frac{N_{o}}{\sqrt{2 \pi \sigma}} \exp \left(-\frac{x^{2}}{2 \sigma^{2}}\right)
$$

$\mathrm{p}(\mathrm{x})=$ densidade populacional $\sigma=$ desvio padrão. $\sigma^{2}=$ variança.

O movimento random walk pode ser representado em um espaço bidimensional. Dessa maneira, se os animais forem colocados no centro das coordenadas, poderemos observar após alguns passos (n) ou algum tempo (t) esse movimento relacionado a uma distribuição normal dimensional conforme a fórmula abaixo (Sharov, 1997):

$$
p(x, y)=\frac{N_{o}}{2 \pi \sigma^{2}} \exp \left(-\frac{x^{2}+y^{2}}{2 \sigma^{2}}\right)
$$

Essa fórmula permite a modelagem do comportamento do animal em relação ao seu deslocamento, predizendo assim, uma possível posição futura de acordo com certo número de passos pré-estabelecidos ou identificados.

\section{MOVIMENTOBROWNIANO}

Os estudos sobre o movimento browniano datam do século XIX, onde o naturalista inglês Robert Brown através de experimentos observou que os grãos de pólen boiavam em um copo de água e que se

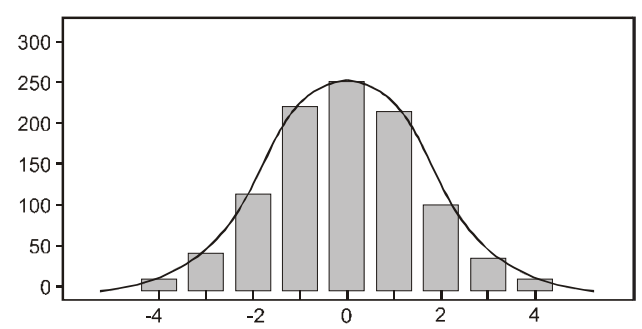

Figura 4. Distribuição normal. (Normal distribution). 


\section{MODELOS RANDOM WALKE BROWNIANO PARA O DESLOCAMENTO BOVINO}

movimentavam constantemente, em um zigue-zague caótico, sem que nenhuma força os empurrasse. O pesquisador chegou a inferir que o mesmo estivesse vivo em função dos movimentos realizados na água, mas verificou em seguida que o mesmo efeito ocorria com um pó de granito e de vidro, descaracterizando desta forma a vida ao pólen (Einstein, 1956).

Tal evento tornou-se uma incógnita para os pesquisadores da época. Não havia nesse início de século nenhuma evidência real de que a matéria fosse composta efetivamente de átomos.

Mas, na realidade esse evento poderia ser explicado através do movimento browniano, o qual foi comprovado pelas experiências realizadas por Albert Einsten em 11 de maio de 1905 através de uma publicação realizada nos Anais de Física daquela época (Einstein, 1956).

O movimento browniano pode ser descrito como um movimento aleatório de partículas macroscópicas em um líquido como conseqüência dos choques das moléculas do líquido sobre as partículas em suspensão.

Um outro ponto importante nessa análise relaciona-se há um padrão escondido nesse movimento aleatório que o classifica como um movimento fractal, pois descreve um padrão dinâmico bem definido. Quem primeiro observou esse evento foi o matemático polonês Benoît Mandelbrot(Nunes, 2006).

Sendo assim, pode-se modelar um sistema ou o comportamento de deslocamento de um animal baseado nessa teoria, para compor uma equação ou um modelo que descreva o comportamento do animal durante o seu deslocamento na busca por água, alimentação ou simplesmente pastagem.

Esses animais ao se deslocarem realização procedimentos muitas vezes tidos como aleatórios, mas existe uma possibilidade de que esses movimentos possam ser desencadeados através de estímulos interiores causadas pela necessidade de realização de alguma tarefa, ou da necessidade de uma adaptação em relação ao meio ambiente em que está inserido, daí a importância de se usar o movimento browniano que é um movimento que permite modelar estas necessidades como condições de contorno do movimento.

\section{METODOLOGIA}

Para a construção e implementação do simulador foi utilizada uma linguagem orientada a objeto $\mathrm{C}++$ da Borland e Linguagem de Metodologia Unificada - UML para a modelagem do Sistema.

A construção do simulador seguiu os princípios descritos por Pressman (2004) através da documentação e análise de modelo e sua posterior modelagem em linguagem UML - Use Case, onde o sistema estava modelado e seus objetos definidos.

Os métodos de cada classe foram concebidos de acordo com a sessão 2 , que referenciou o comportamento social do deslocamento do animal, permitindo assim, definir os parâmetros necessários para a modelagem da classe, em nosso caso, classe BOI.

Em relação a essa mesma sessão, foram fixados alguns elementos que receberam o nome de parâmetros, que são: número de animais em deslocamento, distância de fuga (df), distância social (ds) e número de passos dado pelo BOI líder, para recalculo de novos ângulos.

A figura 5 permite visualizar a tela do simulador e os parâmetros identificados acima.

\section{RESULTADOSEDISCUSSÃO}

Na matemática das probabilidades um processo estocástico pode ser considerado como uma função aleatória. Quando o domínio em que a função está definida é unidimensional (ex. tempo) o processo estocástico é designado por uma série temporal; quando o domínio é de dimensão superior o processo estocástico é designa- 


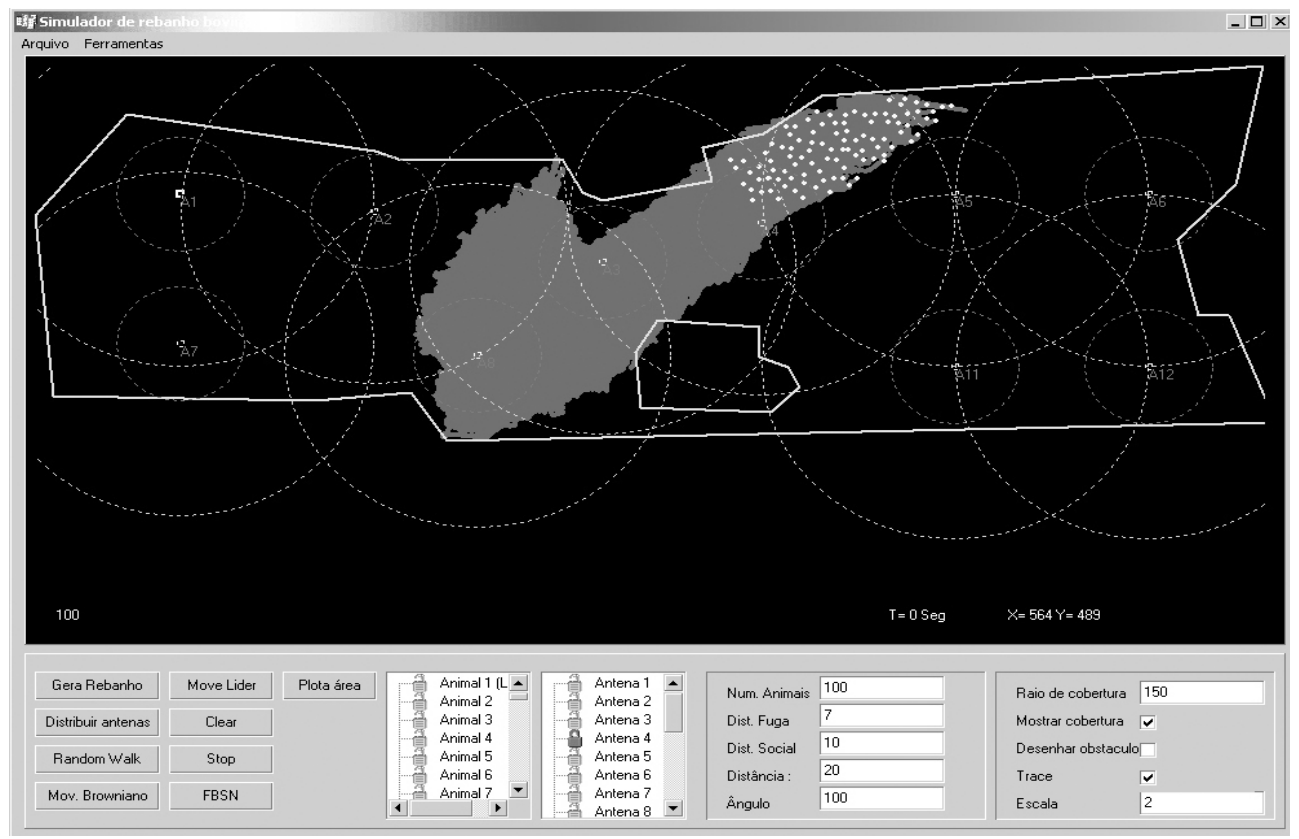

Figura 5. Tela principal do simulador desenvolvido em $C++$ e modelado em UML. (Main screen of the simulator developed in $\mathrm{C}++$ and shaped in $\mathrm{UML}$ ).

do um campo aleatório (Nunes, 2006).

As semelhanças entre esses dois processos são mais do que coincidências. O movimento browniano descreve o movimento de uma partícula flutuando numa superfície de um líquido, recebendo toques de outras partículas no líquido. A partícula é vista como sujeita a uma força aleatória que condiciona o seu movimento.

No caso do random walk considera-se que a partícula toma diferentes caminhos, por passos, e que esses passos são dados em direções aleatórias. O interessante desses processos é que após um percurso suficientemente longo a partícula terá percorrido uma trajetória que tem caráter fractal e que permitirá ao simulador analisar o melhor trajeto (realizado pelo animal) ou a área mais utilizada (Nunes, 2006).

A seguir, será realizado uma comparação entre os movimentos descritos, com o intuito de verificar qual deles prediz melhor o movimento realizado pelo rebanho em seu deslocamento:

O movimento random walk, permite uma maior cobertura em relação as áreas próximas, ou seja, em função da alteração aleatória do seu ângulo e em relação a um determinado número de passos é possível verificar uma maior abrangência nas áreas vizinhas. Esse fato pode ser percebido conforme figura $\mathbf{6}$, obtida do simulador.

É fácil de verificar esse procedimento através da interpretação da figura 6 . Imagine um animal se deslocando de forma aleatória, a cada passo, ele muda a direção de sua trajetória, totalmente ao acaso, variando o seu ângulo aleatoriamente entre $0^{\circ}$ e $360^{\circ}$ e mantendo constante o número de passos ou distância em deslocamento.

$\mathrm{Na}$ figura 7, é possível observar sua variação após um instante $\mathrm{t}+100$. O animal andou um pouco mais, mas, na verdade, se afastou pouco do ponto de sua origem. $\mathrm{O}$

Archivos de zootecnia vol. 58, núm. 223, p. 350. 


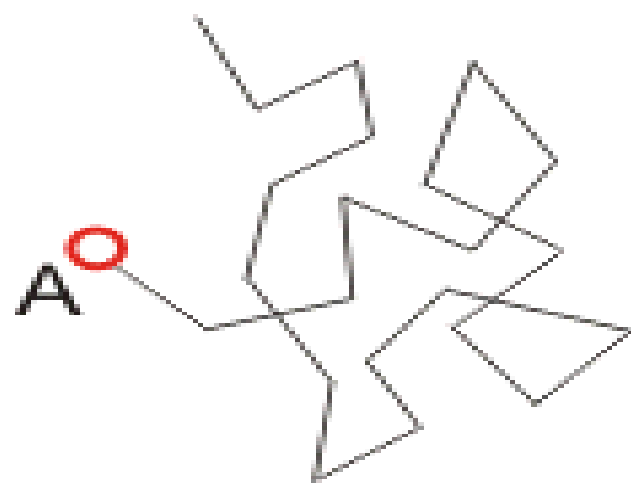

Figura 6. Início dos movimentos. (Begin of the movements).

círculo exterior mostra, mais ou menos, de quanto o animal se afastou do ponto de saída (A). No entanto, é fácil de observar que, depois de um instante $t+100$, ele praticamente continua próximo ao ponto de saída, portanto, ele consegue uma melhor ocupação espacial nas proximidades de sua origem.

Já a figura 8, mostra a situação do animal após $\mathrm{t}+500$. Embora o animal já tenha alcançado uma distância maior em relação a sua origem (círculo exterior), a região cen-

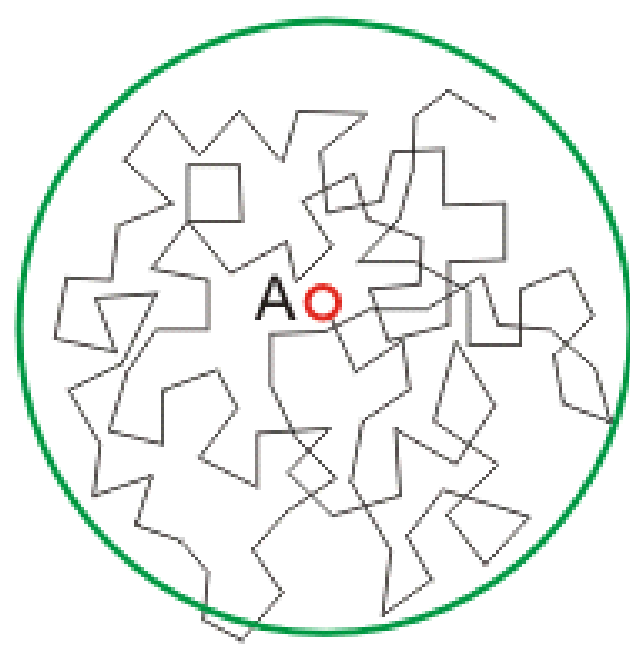

Figura 7. Instante $t+100$. (Instant $t+100)$. tral continua sendo o local mais provável de encontrá-lo.

Conclui-se, portanto, que através do movimento random walk têm-se uma área ou região inicial mais visitada pelo animal, mesmo depois de um tempo $t$ e de um número $n$ de passos. Fica fácil de entender agora, quando é citado que, depois de muito andar, o animal aleatório não se afastou de sua origem.

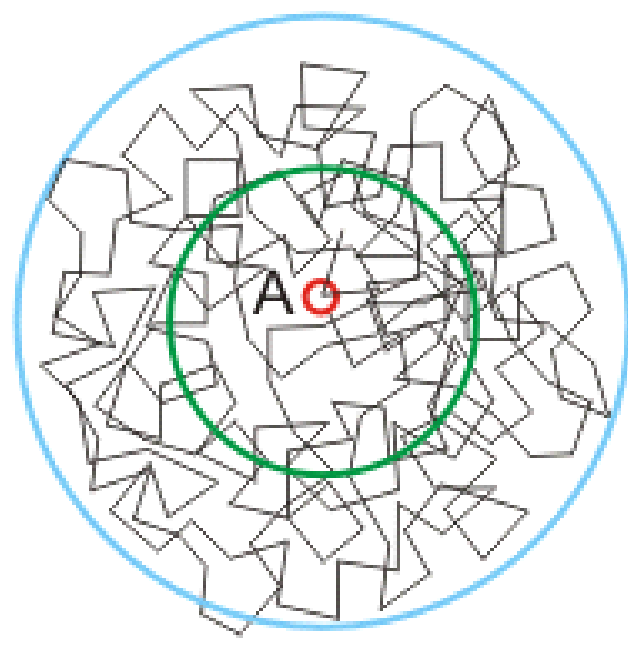

Figura 8. Movimentos realizados após um instante $t+500$. (Movements carried through after a instant $t+500)$.

As figuras 9.1 a 9.6 a seguir, ilustrarão os dados obtidos através do simulador para análise de deslocamento e área de cobertura. O movimento analisado é o random walk, em instantes $t(s)$ :

As figuras ilustradas anteriormente permitem uma boa identificação e compreensão do movimento random walk, pois pode ser percebido que após um $\mathrm{t}(\mathrm{t}>5000)$, o animal tenderá a estar próximo a sua origem, em contra partida, têm-se uma ocupação de espaço na vizinhança bem elevado.

Agora, analisaremos a caminhada através do movimento browniano, o qual também procura descrever um caminho através da interação do animal com o restan- 


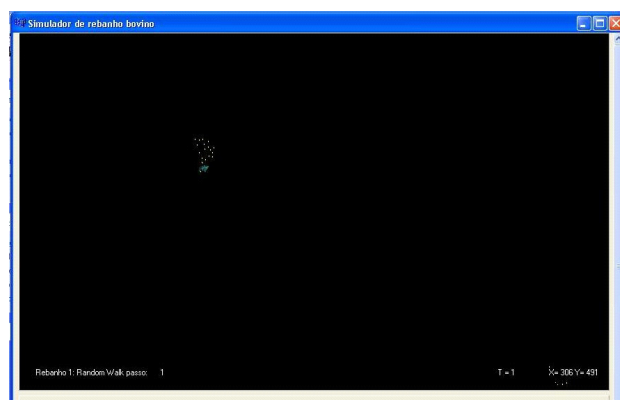

Figura 9.1. Início do movimento random walk $(t=0)$. (Begin of the random walk movement $(\mathrm{t}=0))$

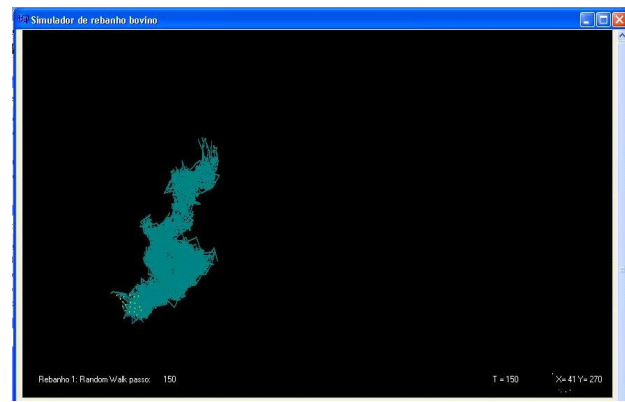

Figura 9.3. Movimento random walk ( $t=$ 300). (Random walk movement $(\mathrm{t}=300)$ ).

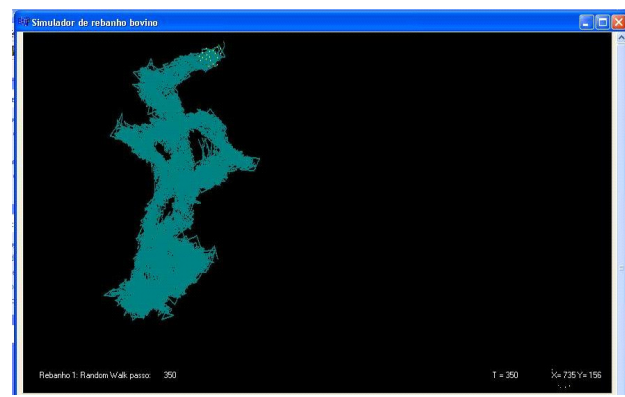

Figura 9.5. Movimento random walk ( $t=$ 700). (Random walk movement $(\mathrm{t}=700)$ ).

te do rebanho e de possíveis obstáculos encontrados durante o percurso, simulando assim, o movimento do animal, com as interações das partículas de água com os grãos de pólen, descritos no artigo de Albert Einstein(Einstein, 1956).

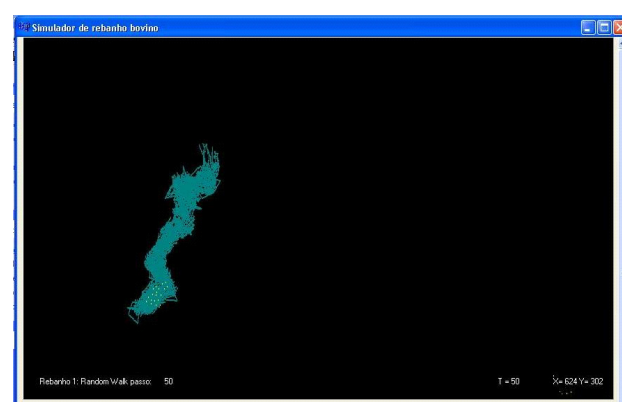

Figura 9.2. Movimento random walk ( $t=$ 100). (Random walk movement $(\mathrm{t}=100)$ ).

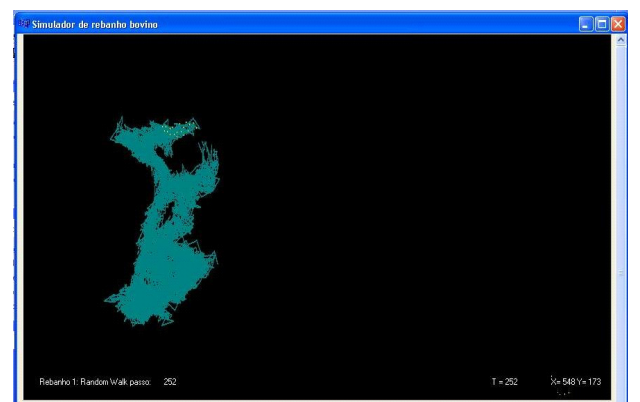

Figura 9.4. Movimento random walk ( $t=$ 500). (Random walk movement $(t=500)$ ).

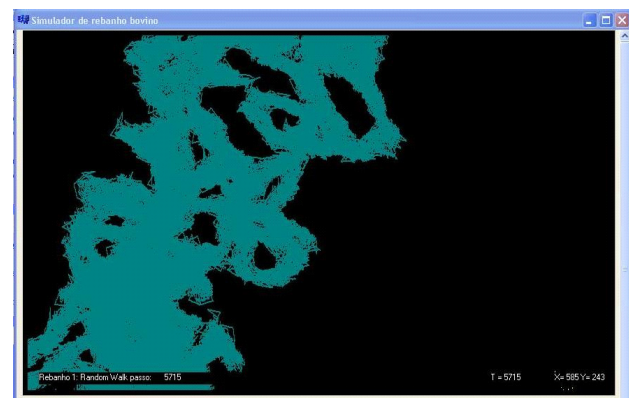

Figura 9.6. Movimento random walk ( $t=$ 5000). (Random walk movement $(\mathrm{t}=5000)$ ).

Assim, pode-se observar que a utilização de um modelo embasado no comportamento browniano permitirá que o animal percorra um espaço muito maior em relação ao random walk, conforme as figuras 10.1 a 10.6 .

Como verificado acima, o movimento 


\section{MODELOS RANDOM WALKE BROWNIANO PARA O DESLOCAMENTO BOVINO}

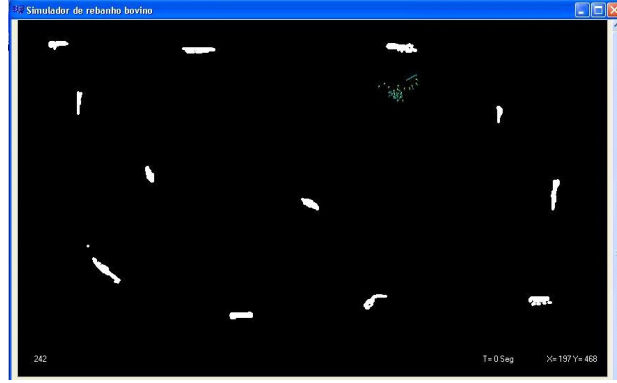

Figura 10.1. Início do movimento browniano $(t=0)$. (Begin of the brownian movement $(t=0)$ ).

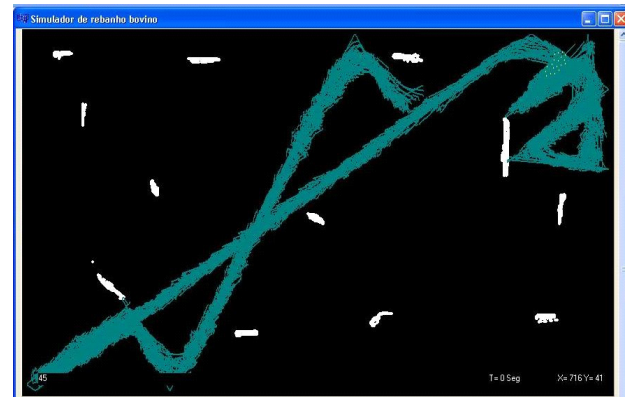

Figura 10.3. Movimento browniano $(t=300)$. (Brownian movement $(\mathrm{t}=300)$ ).

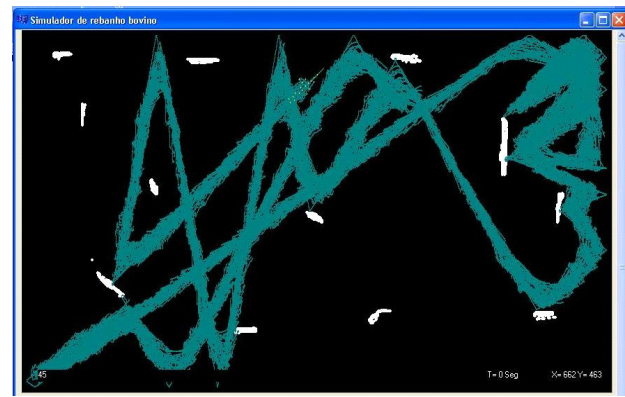

Figura 10.5. Movimento browniano $(t=700)$. (Brownian movement $(\mathrm{t}=700)$ ).

browniano permite uma maior ocupação espacial (figura 10.6 após $\mathrm{t}>5000$ ), uma vez que seu movimento é alterado mediante alguma força externa, necessidade do animal, ou através de alguma obstrução no percurso e forças contrárias ao seu movimento.

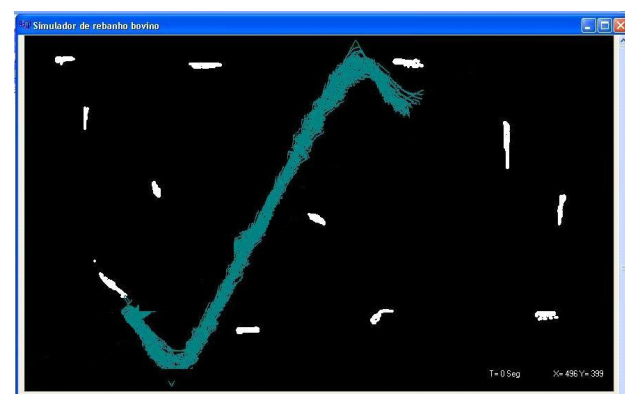

Figura 10.2. Movimento browniano $(t=100)$. (Brownian movement $(\mathrm{t}=100)$ ).

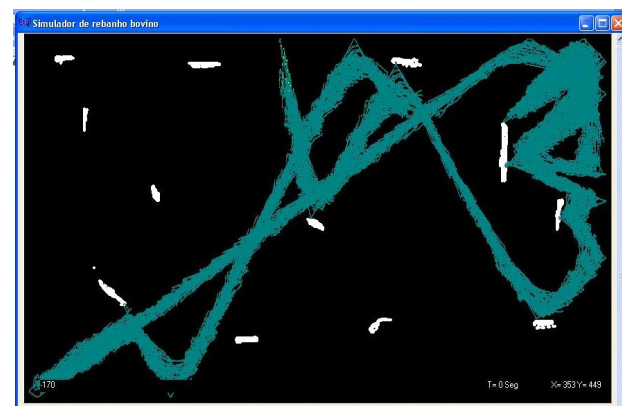

Figura 10.4. Movimento browniano $(t=500)$. (Brownian movement $(\mathrm{t}=500)$ ).

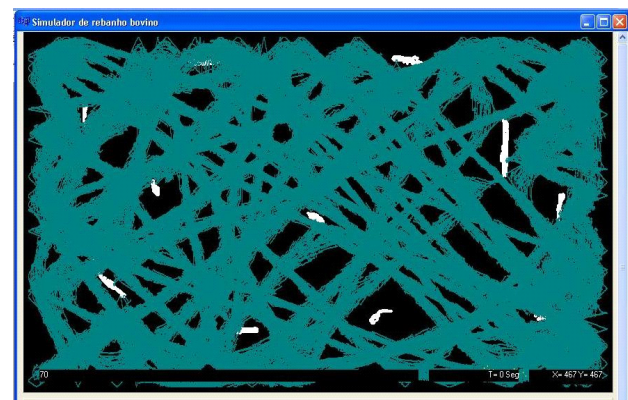

Figura 10.6. Movimento browniano ( $t=$ 5000). (Brownian movement $(\mathrm{t}=5000)$ ).

Espelhando esse conceito em relação à movimentação do rebanho, o animal líder (responsável pelo deslocamento) só alterará o seu caminho mediante alguma necessidade, como, por exemplo, falta de alimentação, água ou, caso encontre algum obstá- 


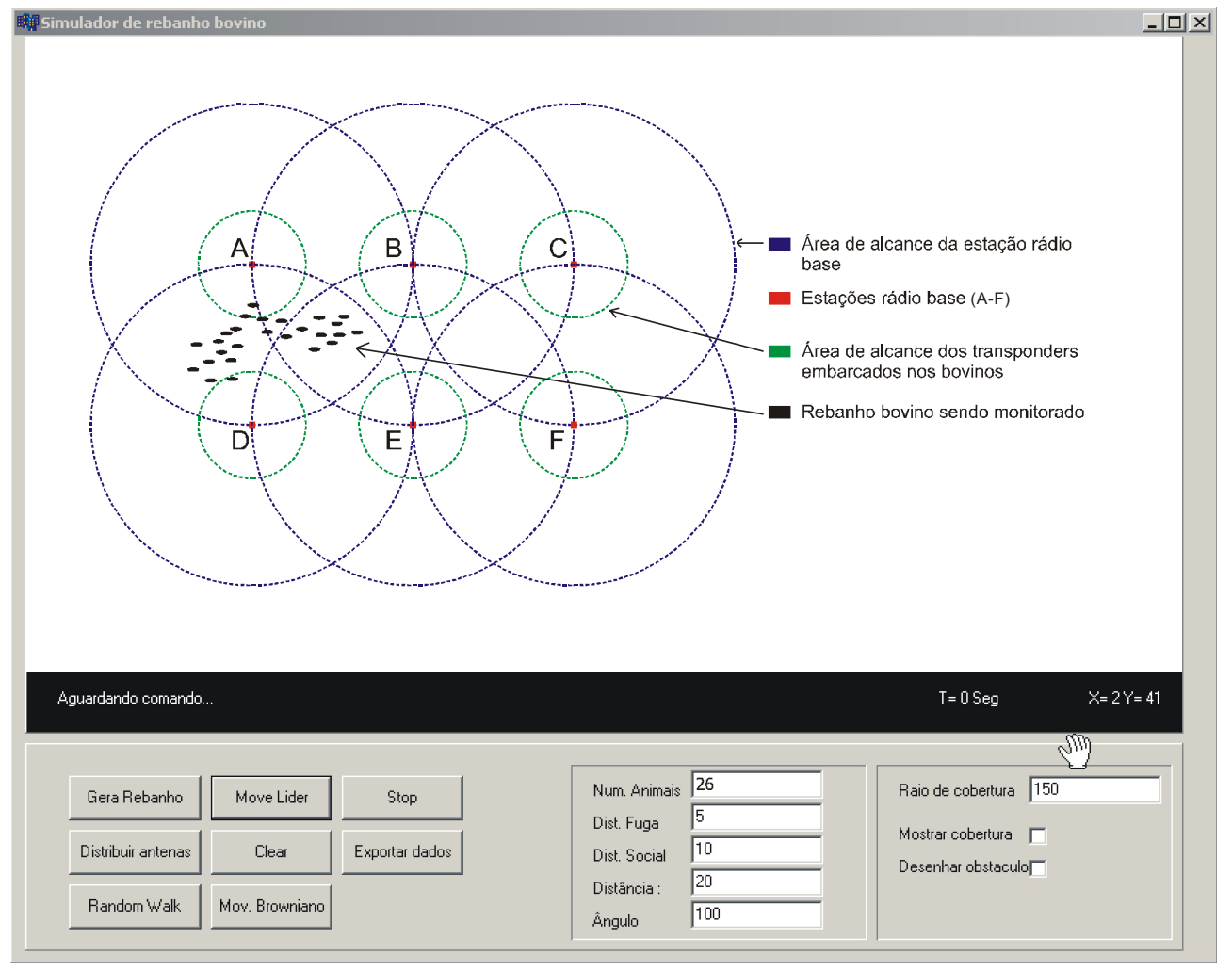

Figura 11. Torres de transmissão para identificação de localização do animal através do protocolo FSBN. (Communications towers for identification of localization of the animal through protocol FSBN).

culo durante o seu percurso, caso contrário, ele descreverá um trajeto aproximadamente linear, até atingir sua necessidade.

\section{APLICAÇÃO do SIMULADOR NO RASTREA- MENTODOREBANHO}

Através da aplicação dos modelos matemáticos utilizados no simulador; random walk e browniano, pode-se descrever a concepção básica do simulador. Foi descrita a partir da análise, do comportamento de deslocamento do animal e do seu posicionamento, permitindo ao gestor analisar e verificar padrões de comportamentos realizados pelo rebanho em seu movimento.

Dessa forma, o simulador pode descrever através de receptores e transmissores os posicionamentos dos animais através de uma rede de sensores sem fio, monitorando os possíveis estados de posição do rebanho, que após a localização ou identi-ficação de um membro, os demais elementos do rebanho também poderão ser identificados através de dispositivos de comunicação sem fio ativo, instalado em alguns animais (Silva et al., 2005).

Através de um desenho bem simples, é possível visualizar o referido modelo e a forma de gerenciamento ou monitoramento dos animais distribuídos em determinadas áreas de pasteio. A figura 11, extraída do simulador em estudo, permite localizar o

Archivos de zootecnia vol. 58, núm. 223, p. 354. 


\section{MODELOS RANDOM WALKE BROWNIANO PARA O DESLOCAMENTO BOVINO}

rebanho através da identificação de um elemento do grupo. Essa figura possui quinze (15) torres de transmissão e recepção com alcance de $150 \mathrm{~m}$, que podem ser identificas pela sigla RB, essas torres além de possuírem comunicação, tem como função disparar sinais para que os dispositivos menores, kits instalados nos animais (BM), como os transponders, para que possam receber o sinal e retransmiti-los para as torres, que se encarregaram de enviar o sinal para o módulo central na sede da fazenda ou outro local qualquer (Silva et al., 2005).

Pode-se gerenciar uma área através de um modelo computacional que poderá inclusive fornecer dados sobre o comportamento individual ou geral de um rebanho, facilitando assim, o trabalho de manejo e demais estudos que se façam necessários.

Outro facilitador do simulador é o de permitir aos zootecnistas e veterinários estudos comparativos, análises de ambiente e interações entre os animais, verificando comportamentos individuais ou em conjuntos durante determinado tempo, de acordo, com a finalidade do estudo.

\section{CONCLUSÕES}

O presente artigo descreveu o modelo

\section{BIBLIOGRAFIA}

Broom, D.M. and K.G. Johnson. 1993. Stress and animal welfare. Chapman and Hall. London.

Broom, D.M. e C.F.M. Molento. 2004. Animal welfare: concept and related issues. Rev. Arch. Vet. Sci., 9: 1-11.

Costa, M.J.R.P. e A.A. Pinto. 2003. Princípios de etologia aplicados ao bem-estar animal. Em: Del Claro, K.; Prezoto, F.E. (Ed.). As distintas faces do comportamento animal. Sociedade Brasileira de Etologia. São Paulo. p. 211-223.

Einstein, A. 1956. Investigations on the theory of the brownian movement. Edited with notes by R. Furth. Translated by A.D. Cowper. Dover Publications, Inc. USA.

Grandin, T. 2000. Principios de comportamiento animal para el manejo de bovinos y otros herbívoros en condiciones extensivas. Publicado de deslocamento de rebanho implementado em um simulador para análise de comportamento do rebanho. Simulando a busca de alimentos, água e mesmo descanso e identificando benefícios relativos ao gerenciamento do rebanho através de um sistema de redes sem fio. Também, pode-se verificar que o movimento browniano é o melhor modelo para simular o deslocamento do rebanho quando comparado com o movimento random walk, principalmente em relação à ocupação de espaço.

Trabalhos futuros usando o simulador para a realização de estudos completos de comportamento do rebanho, através da modelagem e observação de padrões e formas de comportamento, poderão facilitar o cumprimento das normas e regras conforme solicitações internacionais para rastreabilidade do animal, principalmente aqueles com ênfase a exportação, e do próprio SISBOV - Sistema Brasileiro de Identificação e Certificação de Origem Bovina e Bubalina, que é um conjunto de ações, medidas e procedimentos adotados para caracterizar a origem, o estado sanitário, a produção e a produtividade da pecuária nacional e a segurança dos alimentos provenientes dessa exploração econômica.

en: Temple Grandin (comp.) Livestock Handling and Transport. capítulo 5: 63-85. CABI Publishing. Wallingford. Oxon (Reino Unido). Traducción del Dr. Marcos Giménez-Zapiola.

Herrera García, M., F. Peña Blanco y E. Rodero Serrano. 2007. Comportamento Social. Facultad de Veterinaria. Guía Docente Licenciado en veterinaria. Etología y protección animal y etnologia. Tema 13. Curso 2007-2008. Disponível em: http://www.uco.es/organiza/departamentos/prod-animal/economia/aula/img/pictorex/ 06_07_04_TEMA_13.pdf. Acesso em: fevereiro de 2007.

Nadaleto, C.E. y L.J. Marques. 2007. Manejo racional de bovinos. RendCorte consultoria em agronegócio. Disponível em: http://www. rendcorte.com.br/index-\%20Comportamento 
TECH, ARCE, SILVA E COSTA

\%20animal.htm. Acesso em: Março de 2007. Nunes, L.M. 2006. Modelação ambiental. DACT, Departamento de Ambiente e Ciências da Terra. Universidade do Algarve. Disponível: http:// w3.ualg.pt/ Inunes/Pessoal/Disciplinas/Mode lacao-texto.htm. Acesso em 12 de março de 2006.

Pressman, R.S. 2004. Engenharia de software. Makron Books. São Paulo.
Sharov, A. 1997. Quantitative population ecology. Dept. of Entomology, Virginia Tech, Blacksburg. USA. Review appeared in the Plant Pathology Internet Guide Book as a "Tip of the month", August 1997.

Silva, A.C.S., A.C. Arce, S. Souto and E.J.X. Costa 2005. A wireless floating base sensor network for physiological response to livestock. Comput. Electron. Agr., 49: 246-254.

Archivos de zootecnia vol. 58, núm. 223, p. 356. 